\title{
Pesticide exposure and risk of aggressive prostate cancer among private pesticide applicators
}

Larissa A. Pardo ${ }^{1}$, Laura E. Beane Freeman ${ }^{1}$, Catherine C. Lerro ${ }^{1}$, Gabriella Andreotti ${ }^{1}$, Jonathan N. Hofmann ${ }^{1}$, Christine G. Parks², Dale P. Sandler' ${ }^{2}$ Jay H. Lubin ${ }^{3}$, Aaron Blair ${ }^{1}$ and Stella Koutros ${ }^{1,4^{*}}$

\begin{abstract}
Background: Prostate cancer (PCa) is one of the most commonly diagnosed cancers among men in developed countries; however, little is known about modifiable risk factors. Some studies have implicated organochlorine and organophosphate insecticides as risk factors (particularly the organodithioate class) and risk of clinically significant PCa subtypes. However, few studies have evaluated other pesticides. We used data from the Agricultural Health Study, a large prospective cohort of pesticide applicators in North Carolina and lowa, to extend our previous work and evaluate 39 additional pesticides and aggressive PCa.

Methods: We used Cox proportional hazards models, with age as the time scale, to calculate hazard ratios (HRs) and $95 \%$ confidence intervals (Cls) for the association between ever use of individual pesticides and 883 cases of aggressive PCa (distant stage, poorly differentiated grade, Gleason score $\geq 7$, or fatal prostate cancer) diagnosed between 1993 and 2015. All models adjusted for birth year, state, family history of PCa, race, and smoking status. We conducted exposure-response analyses for pesticides with reported lifetime years of use.

Results: There was an increased aggressive PCa risk among ever users of the organodithioate insecticide dimethoate ( $n=54$ exposed cases, $\mathrm{HR}=1.37,95 \% \mathrm{Cl}=1.04,1.80$ ) compared to never users. We observed an inverse association between aggressive PCa and the herbicide triclopyr ( $n=35$ exposed cases, $\mathrm{HR}=0.68,95 \% \mathrm{Cl}=0.48,0.95$ ), with the strongest inverse association for those reporting durations of use above the median ( $\geq 4$ years; $n=13$ exposed cases, $\mathrm{HR}=0.44,95 \% \mathrm{Cl}=0.26,0.77)$.
\end{abstract}

Conclusion: Few additional pesticides were associated with prostate cancer risk after evaluation of extended data from this large cohort of private pesticide applicators.

Keywords: Pesticide, Aggressive prostate cancer (PCa), Organodithioate insecticides, Pesticide applicators

\footnotetext{
*Correspondence: koutross@mail.nih.gov

'Occupational and Environmental Epidemiology Branch, Division of Cancer Epidemiology and Genetics, National Cancer Institute, 9609 Medical Center Drive, Rockville, MD 20850, USA

${ }^{4}$ Occupational and Environmental Epidemiology Branch, Division of Cancer Epidemiology and Genetics, National Cancer Institute, 9609 Medical Center Dr., Room \#6E616, MSC 9771, Bethesda, MD 20892, USA

Full list of author information is available at the end of the article
}

(c) The Author(s). 2020 Open Access This article is licensed under a Creative Commons Attribution 4.0 International License, which permits use, sharing, adaptation, distribution and reproduction in any medium or format, as long as you give appropriate credit to the original author(s) and the source, provide a link to the Creative Commons licence, and indicate if changes were made. The images or other third party material in this article are included in the article's Creative Commons licence, unless indicated otherwise in a credit line to the material. If material is not included in the article's Creative Commons licence and your intended use is not permitted by statutory regulation or exceeds the permitted use, you will need to obtain permission directly from the copyright holder. To view a copy of this licence, visit http://creativecommons.org/licenses/by/4.0/ The Creative Commons Public Domain Dedication waiver (http://creativecommons.org/publicdomain/zero/1.0/) applies to the data made available in this article, unless otherwise stated in a credit line to the data. 


\section{Introduction}

Prostate cancer (PCa) is common among men in developed countries, however, little is known about modifiable risk factors [1]. Investigating potential risk factors for prostate cancer is challenging because incidence rates are affected by $\mathrm{PCa}$ screening. Thus, to avoid potential detection bias, epidemiologic analyses often limit evaluations of prostate cancer to clinically relevant subtypes [2].

Previous epidemiologic studies have linked farming to an increased risk of prostate cancer [3-9]. Analyses of data from the Agricultural Health Study (AHS) revealed a significant excess of both PCa incidence [10] and mortality [11] among pesticide applicators compared to the general population. Exposure to specific individual organochlorine (OC) and organophosphate (OP) insecticides have been linked to prostate cancer in multiple studies [12-16]. Specifically, a previous evaluation in the AHS reported increased risks of aggressive $\mathrm{PCa}$ with exposure to aldrin $(\mathrm{OC})$ as well as the organodithioate class of OP insecticides, including fonofos (OP), terbufos (OP), and malathion (OP) [12]. Other studies reported associations between increased risks of prostate cancer and chlordecone $(\mathrm{OC})[15,16]$ as well as serum metabolite concentrations of chlordane (OC) [13], hexachlorocyclohexanes (OC) [14], and DDT (OC) [14, 16].

We previously published analyses on exposure to 50 commonly reported pesticides used at and before study enrollment and risk of aggressive PCa in the AHS [12]. In the current paper, we use data from the AHS to evaluate possible associations between aggressive $\mathrm{PCa}$ and the use of 39 additional pesticides not previously considered by adding 13 years of follow-up time and 811 additional aggressive PCa cases.

\section{Methods}

\section{Study population and case ascertainment}

The AHS is an ongoing prospective cohort that includes 52,934 licensed private pesticide applicators in Iowa and North Carolina and 4916 licensed commercial applicators in Iowa. The cohort has been described in detail previously [17]. Briefly, the cohort is composed of individuals ( $82 \%$ of the target population enrolled) seeking licenses for pesticides which the U.S. Environmental Protection Agency (EPA) designated restricted use. In the AHS, pesticide exposure information has been collected in 3 phases of questionnaires--Phase 1 (1993-1997), Phase 2 (1999-2003), and Phase 3 (20052010),-with each phase including self-administered questionnaires or a computer assisted telephone interview (CATI) covering demographic, lifestyle, and occupational characteristics. In Phase 1, a total of 57,310 applicators completed the enrollment questionnaire and 25,291 returned the 'Take-Home Applicator' questionnaire. Of those initially enrolled, 36,341 applicators completed the Phase 2 'Pesticide Use Module Applicator' questionnaire, and 24,170 applicators returned the Phase 3 questionnaire (Fig. 1). Full text of all questionnaires is available at https:// aghealth.nih.gov/collaboration/questionnaires.html.

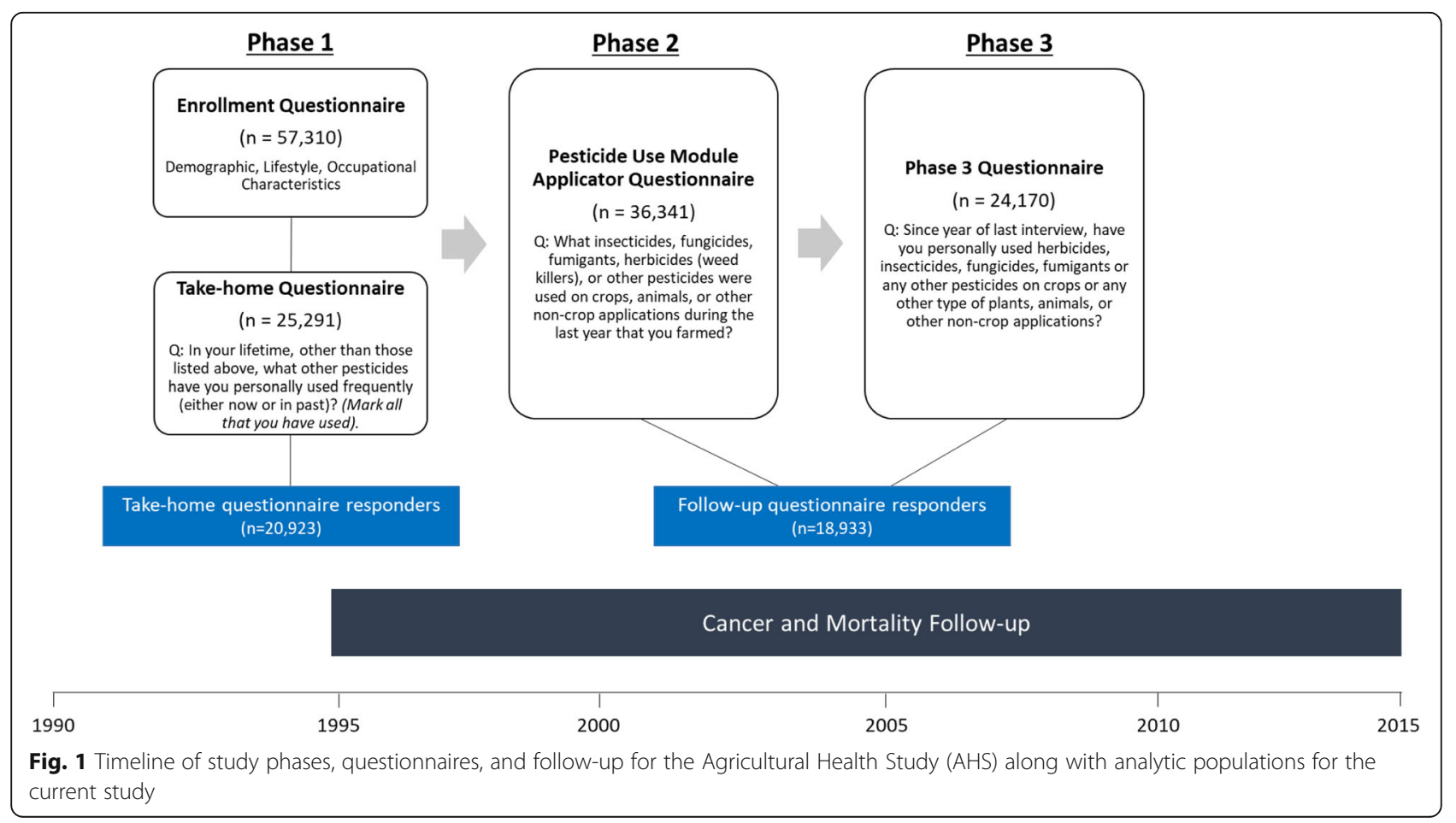


We regularly linked cohort members to state mortality registries and the National Death Index to determine vital status. We also regularly linked participants to cancer registries in Iowa (cases reported through 2015) and North Carolina (cases reported through 2014) to determine incident cancers. Cancers were classified according to the International Classification of Diseases for Oncology, third revision (ICD-O-3) [18]. Aggressive prostate cancer was defined as having one or more of the following tumor characteristics: distant stage, poorly differentiated grade, Gleason score $\geq 7$, or fatal prostate cancer (i.e. underlying cause of death was prostate cancer).

The study protocol, including implied informed consent for completion of questionnaires, was approved by all relevant institutional review boards.

\section{Exposure assessment}

We previously published an analysis of 50 pesticides first reported on the Phase 1 enrollment questionnaire [12]. For the current analysis, we focused on those pesticides that were first reported at take-home (Phase 1 takehome questionnaire) or follow-up (Phase 2 and Phase 3 questionnaires), but not included in prior analyses $(n=$ 39 pesticides). We only evaluated pesticides with 15 or more exposed aggressive PCa cases. Questions regarding pesticide use differed by questionnaire (Fig. 1). The takehome questionnaire provided a checklist of specific pesticide names/active ingredients and applicators marked those that they had ever used. The follow-up questionnaires included open-ended questions and applicators provided the name of the pesticide(s) used. In the Phase 2 questionnaire, applicators reported on use for the last year that they farmed. In the Phase 3 questionnaire, applicators were asked about use since their last questionnaire or interview (which could have been at Phase 1 enrollment, Phase 1 take-home, or Phase 2). The Phase 3 questionnaire also asked applicators to report the lifetime duration (in years) of use for each of the reported pesticides. Since the take-home and follow-up questionnaires were structured differently, this resulted in two different analytic groups: ever/never use to pesticides first reported at takehome (20 pesticides) and ever/never use to pesticides first reported at follow-up (19 pesticides). For pesticides first reported at follow-up (19 pesticides), we also evaluated lifetime duration of use (years), which was ascertained on the Phase 3 questionnaire.

Because many of these pesticides have never been evaluated in the AHS, we also assessed the plausibility of users' duration information (excluding arsenical pesticides). We began by calculating the maximum number of years a pesticide could have been used. This was calculated by subtracting the year a pesticide/active ingredient was first registered in the U.S. from the year the individual completed the phase 3 questionnaire (2005-2010; the year participants responded to questions about duration of use for the given pesticides in the AHS). We used four different sources of information to determine registration year, including the chemical specific Registration Eligibility Decision (RED) published by the U.S. EPA, the EPA's document on "Chemicals Registered for the First Time as Pesticidal Active Ingredients Under FIFRA" [19], different versions of the Farm Chemicals Handbooks [20] covering the timespan of the AHS, and the EXTOXNET: the Extension Toxicology Network [21], which is a Pesticide Information Project of Cooperative Extension Offices of Cornell University, Michigan State University, Oregon State University, and the University of California at Davis. We also evaluated whether any pesticide had been canceled prior to 2010 using the EPA's chemical specific REDs, U.S. Federal Register notices, as well as the Sittig's Handbook of Pesticides and Agricultural Chemicals [22]. We found that fluazifop-butyl and disulfoton had been canceled in the latter halves of 2004 [23] and 2009 [24], respectively. To assess plausibility of duration responses by AHS applicators, we compared the maximum number of years a pesticide could have been available for purchase to selfreported life years of use. We evaluated the frequency and proportion of applicators using a given pesticide as well as the number and proportion of users with reported years of use that were plausible.

\section{Analytic population}

All respondents completed the enrollment questionnaire; however, responses about pesticide use from the take-home and follow-up questionnaires were structured differently resulting in two different analytic groups (Fig. 1). The first analytic group included participants that responded to the take-home questionnaire. Among the 25,291 total participants who completed the takehome questionnaire, we excluded 4368 individuals (2375 commercial applicators [did not receive the Phase 3 follow-up questionnaire], 556 women, 80 who had moved out of state, 620 with prevalent cancer or those diagnosed before completing the questionnaire, and 737 who were diagnosed with non-aggressive $\mathrm{PCa}$ during follow-up), leaving 20,923 private applicators (20,040 non-cases/883 aggressive PCa cases) for analysis. This analytic set was used to evaluate: (1) ever/never use as indicated on the take-home questionnaire, and (2) ever/ never use considering take-home questionnaire use plus information reported at one or both follow-up questionnaire(s) (Phase 2 and/or 3). The second analytic group included participants that responded to both follow-up questionnaires (Phase 2 and Phase 3). Among the 21,142 participants that completed both follow-up questionnaires (Phase 2 and Phase 3), we excluded 2209 
individuals (640 women, 70 who had moved out of state, 963 individuals with prevalent cancer or those diagnosed before completing the Phase 2 or Phase 3 questionnaire, and 536 who were diagnosed with non-aggressive $\mathrm{PCa}$ during follow-up), leaving 18,933 individuals (18,199 non-cases/734 aggressive PCa cases) for analysis.

\section{Statistical analyses}

We used Cox proportional hazards models, with age as the time scale, to calculate hazard ratios (HRs) and 95\% confidence intervals (CIs) for the association between individual pesticide use and aggressive PCa risk. We censored follow-up at the time of aggressive prostate cancer diagnosis, death, movement out of the state, or at December 31, 2014 for North Carolina and 2015 for Iowa, whichever came first. Supplemental Tables 1 and 2 report associations for overall PCa risk. We adjusted all models for year of birth; state (North Carolina, Iowa); family history of prostate cancer in first-degree relatives; race; and cigarette smoking status (never, former, current, missing) based on information on the Phase 1 enrollment questionnaire. Other covariates, including body mass index (BMI: underweight, normal, overweight, obese), fruit servings ( $<1 /$ day, $\geq 1$ /day), and leisure-time physical activity in the winter (none, $>0-2 \mathrm{~h} /$ week, $\geq 3 \mathrm{~h} /$ week), did not materially impact observed point estimates $(\geq 10 \%)$ and, therefore, were not retained in models. We evaluated correlations between ever/never exposure to individual pesticides using the Pearson correlation coefficient and conducted additional adjustment for ever/never use of the three pesticides mostly highly correlated with the pesticide of interest to account for co-exposures (rho range = 0.001-0.2). We also explored adjustment of all models with pesticides previously linked to aggressive $\mathrm{PCa}$ in the AHS (malathion, fonofos, terbufos, and aldrin) [12] as well as with those significantly associated with $\mathrm{PCa}$ in the current analysis (Supplemental Table 3). However, adjustment for co-exposures did not materially impact observed point estimates and were not retained in models. We conducted exposure-response analyses for those pesticides with valid reported duration data from the Phase 3 questionnaire. We created categories for years of use based on the distribution of years reported, split at the median, among all cases. Those with reported years of use that were implausible were coded as having missing exposure in duration analyses. To compute tests for linear trend, the Wald test was used, treating the median value for each category as continuous. We evaluated potential effect modification by family history of PCa. Likelihood ratio tests were used to assess differences between strata.

Using multivariate logistic regression, we also explored possible predictors of prostate specific antigen (PSA) screening including demographic characteristics and individual pesticide use to assess whether screening could explain any observed relationships between pesticides and prostate cancer risk. Participants provided PSA screening (yes/no) information on two follow-up questionnaires $(N=28,880$ men).

All analyses were conducted using SAS, 9.4 (SAS Institute, Cary, North Carolina) and use AHS data release P1REL201701, P2REL201701, and P3REL201701.

\section{Results}

Of the pesticides evaluated in this analysis, acetochlor $(10.8 \%)$ and picloram $(10.6 \%)$ were the most commonly reported herbicides and cyfluthrin was the most commonly reported insecticide (7.6\%) (Table 1$)$. For all pesticides, most applicators reported years of use that were within the plausible range of years of market availability (average percent of applicators reporting plausible years was $97.7 \%$ [range: $88.5-100 \%$ ]). Several pesticides were first registered for use at or after the initial enrollment in the AHS ( $n=7$ on or after 1993) and many others $(n=14)$ within 10 -years prior to enrollment.

The distribution of major prostate cancer risk factors was similar in the two analytic populations in the current analysis as well as compared to the full cohort of private applicators (Supplemental Table 4). The total number of PCa cases was 3169, with aggressive cases making up $54.6 \%$ of all cases.

For pesticides first reported on the take-home questionnaire, there was a significantly increased risk of aggressive $\mathrm{PCa}$ among ever users of dimethoate $(\mathrm{HR}=1.37$, $95 \% \mathrm{CI}=1.04,1.80$ ) compared to never users (Table 2). When we included additional exposure information reported in follow-up questionnaires, the risk for dimethoate was similar $(\mathrm{HR}=1.29,95 \% \mathrm{CI}=0.98,1.70)$. The increased risk for dimethoate was unchanged with additional adjustment for highly correlated pesticides or with any other pesticides linked to $\mathrm{PCa}$ (data not shown). The association between dimethoate use and aggressive $\mathrm{PCa}$ appeared stronger in those with a positive family history of $\mathrm{PCa}(\mathrm{HR}=1.84,95 \% \mathrm{CI}=0.99,3.43)$ compared to those without a family history of $\mathrm{PCa}$ $(\mathrm{HR}=1.31,95 \% \mathrm{CI}=0.96,1.79)$, but the interaction was not statistically significant $\left(P_{\text {interaction }}=0.32\right.$; data not shown). We observed slightly elevated HRs between ever use of bromoxynil ( $\mathrm{HR}=1.17,95 \% \mathrm{CI}=0.99,1.37$ ), linuron $(\mathrm{HR}=1.19,95 \% \mathrm{CI}=0.99,1.42)$, and sethoxydim $(\mathrm{HR}=1.12,95 \% \mathrm{CI}=0.96,1.30)$ and aggressive $\mathrm{PCa}$, but none were statistically significant (Table 2 ). We did not observe any statistically significant interactions between any specific pesticide use reported on the take-home questionnaire and family history of prostate cancer.

For pesticides first reported at Phase 2 and/or Phase 3 follow-ups, there was a significant inverse association between ever use of triclopyr and aggressive $\mathrm{PCa}(\mathrm{HR}=$ $0.68,95 \% \mathrm{CI}=0.48,0.95$ ) (Table 3 ). The risk was not 
Table 1 U.S. registration year and accuracy of self-reported pesticide duration history for 38 chemicals reported on the AHS Phase 3 follow-up questionnaire $(n=24,170)$

\begin{tabular}{|c|c|c|c|c|c|c|c|}
\hline \multirow[t]{2}{*}{ Common name } & \multirow[t]{2}{*}{ Class } & \multirow{2}{*}{$\begin{array}{c}\text { Phase } 1 \\
\text { Take-home } \\
\text { questionnaire }\end{array}$} & \multirow{2}{*}{$\begin{array}{l}\text { Date first } \\
\text { registered } \\
\text { in U.S. }\end{array}$} & \multicolumn{2}{|c|}{$\begin{array}{l}\text { Applicators reported } \\
\text { pesticide use }\end{array}$} & \multicolumn{2}{|c|}{$\begin{array}{l}\text { Users with plausible } \\
\text { responses }{ }^{c}\end{array}$} \\
\hline & & & & $n$ & $\%$ & $\mathrm{n}$ & $\%$ \\
\hline \multicolumn{8}{|l|}{ Herbicide } \\
\hline Acetochlor & Amide & & 1981-1994 & 2604 & 10.8 & 2557 & 98.2 \\
\hline Acifluorfen & Diphenyl ether & $\bullet$ & 1980 & 321 & 1.3 & 317 & 98.8 \\
\hline Bromoxynil & Nitrile & $\bullet$ & 1965 & 679 & 2.8 & 678 & 99.9 \\
\hline Clethodim & Cyclohexene oxime & & 1992 & 545 & 2.3 & 523 & 96.0 \\
\hline Clomazone & Oxazole & $\bullet$ & 1985 & 316 & 1.3 & 307 & 97.2 \\
\hline Clopyralid & Pyridine/Aromatic acid & & 1987 & 1262 & 5.2 & 1252 & 99.2 \\
\hline Cloransulam-methyl & Triazolopyrimidine/Amide & & 1997 & 586 & 2.4 & 554 & 94.5 \\
\hline Dimethenamid & Amide & & 1991 & 748 & 3.1 & 725 & 96.9 \\
\hline Fenoxaprop-p-ethyl & Phenoxy & & 1985 & 551 & 2.3 & 548 & 99.5 \\
\hline Fluazifop-butyl & Phenoxy & $\bullet$ & 1983 & 646 & 2.7 & 638 & 98.8 \\
\hline Flumetsulam & Amide/Triazolopyrimidine & & 1985 & 1095 & 4.5 & 1087 & 99.3 \\
\hline Fomesafen & Amide/Diphenyl ether & & 1987 & 660 & 2.7 & 656 & 99.4 \\
\hline Glufosinate-ammonium & Organophosphorous & & 1993 & 1726 & 7.1 & 1685 & 97.6 \\
\hline Imazaquin & Imidazolinone & $\bullet$ & 1986 & 96 & 0.4 & 94 & 97.9 \\
\hline Isoxaflutole & Oxazole/Cyclopropylisoxazole & & 1998 & 975 & 4.0 & 902 & 92.5 \\
\hline Linuron & Urea & $\bullet$ & 1966 & 48 & 0.2 & 48 & 100.0 \\
\hline Maleic hydrazide & Growth inhibitor/ Gametocides & & 1952 & 387 & 1.6 & 386 & 99.7 \\
\hline Mesotrione & Benzoylcyclohexanedione & & 2001 & 2243 & 9.3 & 1985 & 88.5 \\
\hline Nicosulfuron & Urea & & 1990 & 1693 & 7.0 & 1668 & 98.5 \\
\hline Picloram & Aromatic acid/ Pyridine & & 1964 & 2563 & 10.6 & 2558 & 99.8 \\
\hline Rimsulfuron & Urea & & 1994 & 1146 & 4.7 & 1117 & 97.5 \\
\hline Sethoxydim & Cyclohexene oxime & $\bullet$ & 1982 & 438 & 1.8 & 429 & 97.9 \\
\hline Simazine & Triazine & $\bullet$ & 1957 & 353 & 1.5 & 353 & 100.0 \\
\hline Sodium bentazon & Unclassified & $\bullet$ & 1974/1975-1985 & 382 & 1.6 & 382 & 100.0 \\
\hline Thifensulfuron-methyl & Urea & $\bullet$ & 1989 & 388 & 1.6 & 369 & 95.1 \\
\hline Triclopyr & Pyridine & & 1979 & 1440 & 6.0 & 1416 & 98.3 \\
\hline \multicolumn{8}{|l|}{ Insecticide } \\
\hline Acephate & Organophosphorous & $\bullet$ & 1974 & 1197 & 5.0 & 1145 & 95.7 \\
\hline Bacillus thuringiensis & Unclassified & $\bullet$ & 1961 & 200 & 0.8 & 198 & 99.0 \\
\hline Chloropicrin & Unclassified & $\bullet$ & 1975 & 239 & 1.0 & 223 & 93.3 \\
\hline Cyfluthrin & Pyrethroid & & 1987 & 1849 & 7.6 & 1805 & 97.6 \\
\hline Disulfoton & Organophosphorous & $\bullet$ & 1961 & 270 & 1.1 & 269 & 99.6 \\
\hline Dimethoate & Organophosphorous & $\bullet$ & 1962 & 192 & 0.8 & 192 & 100.0 \\
\hline Endosulfan & Organochlorine & $\bullet$ & 1954 & 203 & 0.8 & 203 & 100.0 \\
\hline Lambda-cyhalothrin & Pyrethroid & & 1989 & 876 & 3.6 & 867 & 99.0 \\
\hline Methomyl & Carbamate & $\bullet$ & 1968 & 162 & 0.7 & 160 & 98.8 \\
\hline Tebupirimfos & Organophosphorous & & 1990-1995 & 1198 & 5.0 & 1148 & 95.8 \\
\hline Tefluthrin & Pyrethroid & $\bullet$ & 1989 & 767 & 3.2 & 733 & 95.6 \\
\hline \multicolumn{8}{|l|}{ Nematicide } \\
\hline 1, 3-dichloropropene & Fumigant & $\bullet$ & 1954 & 158 & 0.7 & 158 & 100.0 \\
\hline
\end{tabular}

${ }^{a}$ Filled circle means chemical first appeared on take-home questionnaire; no circle means chemical first appeared on follow-up questionnaires (Phase 2/3)

${ }^{b}$ Range of years presented for pesticides with evidence of use prior to established registration year; for duration of market availability calculations, the lower

bound of years was used (for those with a range) and rounding to a 5- or 10-year increment was calculated occurred if it fell 1 year short of this increment

'Plausible responses were those where total years reported were $\leq$ number of years calculated between Phase 3 completion year (2005-2010) and the official pesticide registration date 
Table 2 Association between pesticide (ever/never use) and aggressive PCa for those pesticides first reported at the Phase 1 take-home (TH) questionnaire in the Agricultural Health Study (AHS)

\begin{tabular}{|c|c|c|c|c|c|c|}
\hline \multirow[t]{2}{*}{$\begin{array}{l}\text { Common } \\
\text { name }\end{array}$} & \multicolumn{3}{|c|}{$\begin{array}{l}\text { Phase } 1 \text { take-home questionnaire only } \\
\qquad(n=20,923)\end{array}$} & \multicolumn{3}{|c|}{$\begin{array}{l}\text { Phase } 1 \text { take-home questionnaire including follow-up } \\
\text { (Phase 2/Phase } 3 \text { questionnaire) exposure information }\end{array}$} \\
\hline & Non-case & Aggressive PCa & $\mathrm{HR}^{\mathrm{a}}(95 \% \mathrm{Cl})$ & Non-case & Aggressive PCa & $H^{a}(95 \% C l)$ \\
\hline \multicolumn{7}{|l|}{ Herbicide } \\
\hline \multicolumn{7}{|l|}{ Acifluorfen } \\
\hline Never use & 16,626 & 744 & 1 & 13,371 & 621 & 1 \\
\hline Ever use & 3414 & 139 & $1.08(0.90,1.30)$ & 3642 & 146 & $1.06(0.89,1.27)$ \\
\hline \multicolumn{7}{|l|}{ Bromoxynil } \\
\hline Never use & 14,609 & 628 & 1 & 11,557 & 523 & 1 \\
\hline Ever use & 5431 & 255 & $1.17(0.99,1.37)$ & 5758 & 266 & $1.14(0.96,1.34)$ \\
\hline \multicolumn{7}{|l|}{ Clomazone } \\
\hline Never use & 16,850 & 756 & 1 & 13,471 & 632 & 1 \\
\hline Ever use & 3190 & 127 & $0.96(0.79,1.17)$ & 3462 & 132 & $0.94(0.78,1.14)$ \\
\hline \multicolumn{7}{|l|}{ Fluazifop-butyl } \\
\hline Never use & 16,682 & 750 & 1 & 13,077 & 620 & 1 \\
\hline Ever use & 3358 & 133 & $1.07(0.89,1.29)$ & 3909 & 149 & $1.01(0.84,1.21)$ \\
\hline \multicolumn{7}{|l|}{ Imazaquin } \\
\hline Never use & 17,052 & 770 & 1 & 13,880 & 646 & 1 \\
\hline Ever use & 2988 & 113 & $0.99(0.81,1.20)$ & 3101 & 116 & $0.99(0.81,1.20)$ \\
\hline \multicolumn{7}{|l|}{ Linuron } \\
\hline Never use & 17,509 & 742 & 1 & 14,267 & 622 & 1 \\
\hline Ever use & 2531 & 141 & $1.19(0.99,1.42)$ & 2548 & 143 & $1.19(0.996,1.43)$ \\
\hline \multicolumn{7}{|l|}{ Sethoxydim } \\
\hline Never use & 14,404 & 647 & 1 & 11,486 & 546 & 1 \\
\hline Ever use & 5636 & 236 & $1.12(0.96,1.30)$ & 5835 & 239 & $1.07(0.91,1.24)$ \\
\hline \multicolumn{7}{|l|}{ Simazine } \\
\hline Never use & 18,454 & 803 & 1 & 14,922 & 671 & 1 \\
\hline Ever use & 1586 & 80 & $1.19(0.94,1.51)$ & 1724 & 86 & $1.17(0.93,1.48)$ \\
\hline \multicolumn{7}{|c|}{ Sodium bentazon } \\
\hline Never use & 12,911 & 573 & 1 & 10,250 & 476 & 1 \\
\hline Ever use & 7129 & 310 & $1.08(0.93,1.25)$ & 7332 & 317 & $1.06(0.91,1.23)$ \\
\hline \multicolumn{7}{|c|}{ Thifensulfuron-methyl } \\
\hline Never use & 18,108 & 821 & 1 & 14,355 & 668 & 1 \\
\hline Ever use & 1932 & 62 & $0.91(0.70,1.18)$ & 2389 & 83 & $1.00(0.79,1.26)$ \\
\hline \multicolumn{7}{|l|}{ Insecticide } \\
\hline \multicolumn{7}{|l|}{ Acephate } \\
\hline Never use & 18,069 & 811 & 1 & 14,377 & 671 & 1 \\
\hline Ever use & 1971 & 72 & $1.06(0.82,1.39)$ & 2427 & 85 & $1.00(0.77,1.29)$ \\
\hline \multicolumn{7}{|c|}{ Bacillus thuringiensis } \\
\hline Never use & 18,256 & 813 & 1 & 14,855 & 689 & 1 \\
\hline Ever use & 1784 & 70 & $1.00(0.77,1.29)$ & 1904 & 71 & $0.93(0.72,1.20)$ \\
\hline \multicolumn{7}{|l|}{ Chloropicrin } \\
\hline Never use & 19,644 & 865 & 1 & 15,944 & 730 & 1 \\
\hline Ever use & 396 & 18 & $1.48(0.92,2.38)$ & 566 & 20 & $1.21(0.77,1.91)$ \\
\hline
\end{tabular}


Table 2 Association between pesticide (ever/never use) and aggressive PCa for those pesticides first reported at the Phase 1 take-home (TH) questionnaire in the Agricultural Health Study (AHS) (Continued)

\begin{tabular}{|c|c|c|c|c|c|c|}
\hline \multirow[t]{2}{*}{$\begin{array}{l}\text { Common } \\
\text { name }\end{array}$} & \multicolumn{3}{|c|}{$\begin{array}{l}\text { Phase } 1 \text { take-home questionnaire only } \\
\qquad(n=20,923)\end{array}$} & \multicolumn{3}{|c|}{$\begin{array}{l}\text { Phase } 1 \text { take-home questionnaire including follow-up } \\
\text { (Phase 2/Phase } 3 \text { questionnaire) exposure information }\end{array}$} \\
\hline & Non-case & Aggressive $\mathrm{PCa}$ & $\mathrm{HR}^{\mathrm{a}}(95 \% \mathrm{Cl})$ & Non-case & Aggressive $\mathrm{PCa}$ & $\mathrm{HR}^{\mathrm{a}}(95 \% \mathrm{Cl})$ \\
\hline \multicolumn{7}{|l|}{ Disulfoton } \\
\hline Never use & 18,332 & 815 & 1 & 14,927 & 684 & 1 \\
\hline Ever use & 1708 & 68 & $0.96(0.74,1.26)$ & 1807 & 72 & $0.96(0.74,1.25)$ \\
\hline \multicolumn{7}{|l|}{ Dimethoate } \\
\hline Never use & 19,262 & 829 & 1 & 15,673 & 695 & 1 \\
\hline Ever use & 778 & 54 & $1.37(1.04,1.80)$ & 870 & 55 & $1.29(0.98,1.70)$ \\
\hline \multicolumn{7}{|l|}{ Endosulfan } \\
\hline Never use & 19,407 & 860 & 1 & 15,777 & 722 & 1 \\
\hline Ever use & 633 & 23 & $0.85(0.56,1.30)$ & 750 & 25 & $0.78(0.51,1.17)$ \\
\hline \multicolumn{7}{|l|}{ Methomyl } \\
\hline Never use & 18,532 & 827 & 1 & 15,133 & 698 & 1 \\
\hline Ever use & 1508 & 56 & $1.05(0.78,1.40)$ & 1597 & 58 & $1.03(0.77,1.37)$ \\
\hline \multicolumn{7}{|l|}{ Tefluthrin } \\
\hline Never use & 18,635 & 828 & 1 & 14,805 & 684 & 1 \\
\hline Ever use & 1405 & 55 & $0.99(0.75,1.31)$ & 1855 & 71 & $0.98(0.76,1.25)$ \\
\hline \multicolumn{7}{|l|}{ Nematicide } \\
\hline \multicolumn{7}{|c|}{ 1, 3-dichloropropene } \\
\hline Never use & 19,172 & 848 & 1 & 15,669 & 716 & 1 \\
\hline Ever use & 868 & 35 & $1.14(0.80,1.62)$ & 944 & 37 & $1.11(0.78,1.57)$ \\
\hline \multicolumn{7}{|l|}{ Other } \\
\hline \multicolumn{7}{|c|}{ Arsenical pesticides ${ }^{c}$} \\
\hline Never use & 19,309 & 828 & 1 & - & - & - \\
\hline Ever use & 731 & 55 & $1.17(0.88,1.55)$ & - & - & - \\
\hline
\end{tabular}

a Using age as the time metric and adjusted for state, birth year, family history of PCa, race, and smoking status

${ }^{b}$ Numbers add to less than total number of take-home responders $(n=20,923)$ due to missing responses for follow-up use information

${ }^{c}$ Arsenical pesticides consist of lead arsenate (insecticide), organic arsenic (herbicide), and inorganic arsenic (herbicide); only reported use reported on

take-home questionnaire

impacted by additional adjustment for other highly correlated pesticides or with any other pesticide linked to PCa (data not shown). Exposure-response analyses evaluating years of use of triclopyr showed that the HR was lowest among those who reported using triclopyr $\geq$ 4 years compared to never users $(\mathrm{HR}=0.44,95 \% \mathrm{CI}=$ $0.26,0.77, n=13$ exposed cases, $p$-value for trend $=$ 0.004 , Table 4). We observed no other positive or inverse associations between ever use or duration of use of other pesticides first reported at follow-up and aggressive PCa (Tables 3 and 4).

Supplemental Table 5 shows various predictors of PSA screening. Eighty-one percent of all men $(23,416 / 28,880)$ and $87 \%$ of men over the age of $50(22,217 / 25,421)$ had received a PSA test (Supplemental Table 5). Nearly all PCa cases were screened for PSA (overall PCa: 92.6\%, aggressive PCa: 91.7\%;). State, family history, age, marriage status, smoking status, educational attainment, and
BMI were all predictors of PSA. Neither dimethoate nor triclopyr were associated with PSA testing, after controlling for other predictors.

\section{Discussion}

This study reports on the risk of aggressive PCa in relation to use of 39 individual pesticides that have not been previously evaluated in a large prospective cohort of private pesticide applicators. We found no association between the use of most of these pesticides and risk of aggressive prostate cancer. However, we did find a significantly elevated risk of aggressive PCa among ever users of the OP insecticide dimethoate and an inverse association between ever use of the herbicide triclopyr. We also found suggestive associations with commonly used herbicides such as bromoxynil, linuron, and sethoxydim and aggressive $\mathrm{PCa}$. 
Table 3 Association between pesticide (ever/never use) and aggressive PCa for those pesticides first reported at follow-up (Phase 2 and Phase 3 questionnaires) in the Agricultural Health Study (AHS)

\begin{tabular}{ll}
\hline Common name & \multicolumn{2}{c}{ Follow-up questionnaire (Phase 2 and Phase 3$)^{\mathrm{b}}$} \\
\cline { 2 - 3 } & Non-case $\quad$ Aggressive PCa $\quad \operatorname{HR}^{\mathrm{a}}(95 \% \mathrm{Cl})$
\end{tabular}

\section{Herbicide}

Acetochlor

Never use $\quad 15,110 \quad 640$

$\begin{array}{lll}\text { Ever use } \quad 3089 & 94\end{array}$

Clethodim

Never use $\quad 17,526 \quad 717$

Ever use $\quad 673$

Clopyralid

Never use $\quad 16,513 \quad 690$

Ever use $\quad 1686$

Cloransulam-methyl

Never use $\quad 17,453$

Ever use $\quad 746$

Dimethenamid

Never use $\quad 17,238$

Ever use $\quad 961$

Fenoxaprop-p-ethyl

Never use $\quad 17,228$

Ever use $\quad 97$

Flumetsulam

Never use $\quad 16,612$

Ever use $\quad 1587$

Fomesafen

Never use $\quad 16,955$

1244

Ever use

Glufosinate-ammonium
Never use $\quad 16,538$

Ever use

1661

Isoxaflutole

Never use

17,172

Ever use

1027

Maleic hydrazide

Never use $\quad 17,326$

Ever use

873

Mesotrione

Never use $\quad 16,312$

1887

Ever use

Nicosulfuron

Never use

15,931

2268

640

94

17

90

44

709

25

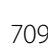

25

703

31

687

47

690

44

692

42

703

31

709

25

692

42

674

60
$0.91(0.67,1.23)$
Table 3 Association between pesticide (ever/never use) and aggressive PCa for those pesticides first reported at follow-up (Phase 2 and Phase 3 questionnaires) in the Agricultural Health Study (AHS) (Continued)

\begin{tabular}{|c|c|c|c|}
\hline \multirow[t]{2}{*}{ Common name } & \multicolumn{3}{|c|}{$\begin{array}{l}\text { Follow-up questionnaire (Phase } 2 \text { and Phase } 3)^{b} \\
\qquad N=18,933\end{array}$} \\
\hline & Non-case & Aggressive PCa & $\mathrm{HR}^{\mathrm{a}}(95 \% \mathrm{Cl})$ \\
\hline \multicolumn{4}{|l|}{ Picloram } \\
\hline Never use & 15,848 & 652 & 1 \\
\hline Ever use & 2351 & 82 & $0.97(0.77,1.23)$ \\
\hline \multicolumn{4}{|l|}{ Rimsulfuron } \\
\hline Never use & 16,728 & 694 & 1 \\
\hline Ever use & 1471 & 40 & $0.83(0.60,1.15)$ \\
\hline \multicolumn{4}{|l|}{ Triclopyr } \\
\hline Never use & 16,788 & 699 & 1 \\
\hline Ever use & 1411 & 35 & $0.68(0.48,0.95)$ \\
\hline \multicolumn{4}{|l|}{ Insecticide } \\
\hline \multicolumn{4}{|l|}{ Cyfluthrin } \\
\hline Never use & 16,399 & 691 & 1 \\
\hline Ever use & 1800 & 43 & $0.81(0.59,1.10)$ \\
\hline \multicolumn{4}{|c|}{ Lambda-cyhalothrin } \\
\hline Never use & 17,257 & 712 & 1 \\
\hline Ever use & 942 & 22 & $0.96(0.63,1.47)$ \\
\hline \multicolumn{4}{|l|}{ Tebupirimfos } \\
\hline Never use & 17,036 & 702 & 1 \\
\hline Ever use & 1163 & 32 & $0.86(0.60,1.23)$ \\
\hline
\end{tabular}

a Using age as the time metric and adjusted for state, birth year, family history of $\mathrm{PCa}$, race, and smoking status

${ }^{\mathrm{b}}$ Numbers may not add up to total, due to missing responses for pesticides

Dimethoate (O,O-dimethyl S-methylcarbamoylmethyl phosphorodithioate) is a dithioate OP insecticide. It was first registered for use in the U.S. in 1962 for use on alfalfa, wheat, cotton, and corn [25]. In 1983, the International Agency for Research on Cancer (IARC) failed to classify dimethoate regarding human carcinogenicity due to lack of available data [26]. In 1991, the U.S. EPA classified dimethoate as a possible human carcinogen (Group C). Dimethoate exhibited carcinogenic effects in some strains of rats and mice, with neoplasms occurring in the endocrine organs, liver, and lymphatic systems [27]; however, there have been no links to prostate cancer in humans. In this analysis, we observed a higher risk for aggressive $\mathrm{PCa}$ among applicators who reported dimethoate use. Pesticide applicators who reported use of dimethoate were more likely to report application methods related to animals, which is also consistent with known uses for dimethoate [28]. Our inclusion of information on pesticide use during follow-up yielded a similar magnitude of elevated risk, although the association was no longer statistically significant. This may be due to a decrease in sample size from non-response in the 
Table 4 Association between duration of pesticide use (in years) and aggressive PCa for those pesticides first reported at follow-up (Phase 2 and Phase 3 questionnaires) in the Agricultural Health Study $(\mathrm{AHS})^{\text {b }}$

\begin{tabular}{|c|c|c|c|}
\hline Common name & Non-case & Aggressive $\mathrm{PCa}$ & HR $(95 \% \mathrm{Cl})^{\mathrm{a}}$ \\
\hline \multicolumn{4}{|l|}{ Herbicide } \\
\hline \multicolumn{4}{|l|}{ Acetochlor } \\
\hline Never use & 15,097 & 633 & 1 \\
\hline$<9$ years & 1213 & 28 & $0.76(0.52,1.12)$ \\
\hline$\geq 9$ years & 882 & 30 & $1.12(0.77,1.63)$ \\
\hline p-trend & 0.96 & & \\
\hline \multicolumn{4}{|l|}{ Clopyralid } \\
\hline Never use & 16,504 & 684 & 1 \\
\hline$<5$ years & 469 & 9 & $0.66(0.34,1.28)$ \\
\hline$\geq 5$ years & 565 & 15 & $0.88(0.53,1.47)$ \\
\hline p-trend & 0.39 & & \\
\hline \multicolumn{4}{|c|}{ Glufosinate-ammonium } \\
\hline Never use & 16,516 & 683 & 1 \\
\hline$<5$ years & 613 & 12 & $0.67(0.38,1.20)$ \\
\hline$\geq 5$ years & 526 & 16 & $1.03(0.62,1.70)$ \\
\hline p-trend & 0.79 & & \\
\hline \multicolumn{4}{|l|}{ Mesotrione } \\
\hline Never use & 16,284 & 680 & 1 \\
\hline$<4$ years & 753 & 17 & $0.82(0.50,1.33)$ \\
\hline$\geq 4$ years & 856 & 17 & $0.77(0.48,1.26)$ \\
\hline $\mathrm{p}$-trend & 0.22 & & \\
\hline \multicolumn{4}{|l|}{ Nicosulfuron } \\
\hline Never use & 15,921 & 668 & 1 \\
\hline$<5$ years & 560 & 15 & $0.88(0.53,1.48)$ \\
\hline$\geq 5$ years & 820 & 21 & $0.84(0.54,1.30)$ \\
\hline p-trend & 0.39 & & \\
\hline \multicolumn{4}{|l|}{ Picloram } \\
\hline Never use & 15,817 & 637 & 1 \\
\hline$<6$ years & 899 & 32 & $1.15(0.80,1.65)$ \\
\hline$\geq 6$ years & 1145 & 32 & $0.79(0.55,1.14)$ \\
\hline $\mathrm{p}$-trend & 0.28 & & \\
\hline \multicolumn{4}{|l|}{ Rimsulfuron } \\
\hline Never use & 16,717 & 690 & 1 \\
\hline$<4$ years & 333 & 10 & $1.00(0.54,1.88)$ \\
\hline$\geq 4$ years & 573 & 14 & $0.78(0.46,1.32)$ \\
\hline p-trend & 0.39 & & \\
\hline \multicolumn{4}{|l|}{ Triclopyr } \\
\hline Never use & 16,770 & 690 & 1 \\
\hline$<4$ years & 282 & 10 & $0.99(0.53,1.85)$ \\
\hline$\geq 4$ years & 848 & 13 & $0.44(0.26,0.77)$ \\
\hline p-trend & 0.004 & & \\
\hline
\end{tabular}

Table 4 Association between duration of pesticide use (in years) and aggressive PCa for those pesticides first reported at follow-up (Phase 2 and Phase 3 questionnaires) in the Agricultural Health Study (AHS) ${ }^{\mathrm{b}}$ (Continued)

\begin{tabular}{llll}
\hline Common name & Non-case & Aggressive PCa & $\mathrm{HR}(95 \% \mathrm{Cl})^{\mathrm{a}}$ \\
\hline $\begin{array}{c}\text { Insecticide } \\
\text { Cyfluthrin }\end{array}$ & & & \\
$\quad$ Never use & 16,380 & 686 & 1 \\
$<6$ years & 803 & 17 & $0.71(0.44,1.15)$ \\
$\geq 6$ years & 645 & 14 & $0.82(0.48,1.39)$ \\
p-trend & 0.22 & & \\
$\begin{array}{l}\text { Tebupirimfos } \\
\text { Never use }\end{array}$ & 17,022 & 698 & 1 \\
$<6$ years & 540 & 12 & $0.69(0.39,1.23)$ \\
$\geq 6$ years & 381 & 12 & $1.09(0.61,1.93)$ \\
p-trend & 0.67 & & \\
\hline
\end{tabular}

a Using age as the time metric and adjusted for state, birth year, family history of $\mathrm{PCa}$, race, and smoking status

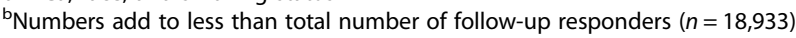
due to missing responses for duration of use information

follow-up questionnaires. Exposure-response analyses for duration of dimethoate use were also precluded due to a lack of use during follow-up for this insecticide (primarily historical use). Work practice information from pesticide applicators who used dimethoate suggested that variations in application methods and use of personal protective equipment did not materially change the observed association between dimethoate and aggressive prostate cancer (data not shown).

To our knowledge, no previous epidemiologic studies have reported on the specific relationship between dimethoate and $\mathrm{PCa}$ (or any other cancer site); however, some studies found associations between aggressive PCa risk and exposure to other dithioate insecticides, including fonofos, malathion, terbufos, and azinphos-methyl $[12,29]$. The mechanism of pesticidal action of OP insecticides is to inhibit the enzyme that breaks down the neurotransmitter acetylcholine; however, this mechanism has not been explicitly linked to prostate cancer risk. Recently, a large genomic analysis of prostate cancer in 140,000 men found that genetic variants in pathways related to neurotransmission release were enriched in prostate cancer [30]. We also have suggested that pesticides may interact with genetic variants in signal transduction and cellular communication pathways affected by neurotransmission [31]. Thus, a link between acetylcholinesterase inhibition and prostate cancer is plausible. These findings indicate the need for future work on OP insecticides (specifically organodithioate insecticides) to identify a biologically plausible link to prostate cancer.

We also found an inverse association between triclopyr use and aggressive PCa. Exposure-response analyses, 
using lifetime years of use among those who completed the Phase 3 questionnaire showed that increasing years of use was significantly associated with a decreased risk of prostate cancer ( $\mathrm{p}$-trend $=0.008$ ). In 1995, the U.S. EPA deemed triclopyr not classifiable as to human carcinogenicity (Group D) because no epidemiologic studies had been conducted and only suggestive results had been seen in animal studies [32]. Triclopyr (3,5,6-trichloro-2-pyridyloxyacetic acid) is a pyridine herbicide, which breaks down into 3,5,6-trichloro-2-pyridinol (TCPy) in the soil. Studies have suggested that TCPy can persist for a maximum of 9 months, but most soil samples exhibited a half-life of less than 90 days [33]. There have been some epidemiologic studies evaluating the association between the TCPy-also a metabolite of the insecticide chlorpyrifos-and sex and thyroid hormones [34-36]. These studies observed decreased levels of testosterone [34], estradiol [33], and altered levels of thyroid function markers, several of which have been suspected in the etiology of prostate cancer [37-43]. However, in humans, triclopyr is rapidly eliminated with more than $80 \%$ unchanged when excreted in urine, thus, it is unclear if there would be hormonal effects in humans from TCPy exposure [44]. There are no direct data about possible endocrine disrupting properties of triclopyr and alternative explanations including the possibility of a chance finding cannot be ruled out. This pesticide, however, is commonly mixed with suspected endocrine disrupting chlorophenoxy herbicides $[45,46]$ because of a common mechanism of action (triclopyr is the pyridine analogue of 2,4,5- trichlorophenoxyacetic acid).

There were small increased risks of aggressive PCa from reported use of the herbicides bromoxynil, linuron, and sethoxydim, but none were statistically significant. Bromoxynil, or bromoxynil phenol, was classified by the U.S. EPA as a possible human carcinogen (Group C) in 1998 based on only a few studies conducted in animals [47]. To our knowledge, there have been no human studies published on the health effects of bromoxynil. Linuron, or 3-(3,4dichlorophenyl)-1-methoxy-1-methylurea, was classified by the U.S. EPA as a possible human carcinogen (Group C) in 1990 due to observed testicular effects in rats, including interstitial cell hyperplasia and adenomas [48]. In rats, linuron is considered to play a toxic role in the male reproductive system as it has been indicated to significantly alter the expression of genes associated with testosterone synthesis, cell proliferation, and apoptosis [49]. Sethoxydim, or 2[1-(ethoxyimino)butyl]-5-[2-(ethylthio)propyl]-3-hydroxy2-cyclohexen-1-one, was classified by the U.S. EPA in 2005 as not likely to be carcinogenic in humans based on the lack of evidence of carcinogenicity in rats and mice [50]. More data in humans are needed to adequately understand the relationship between exposure to these chemicals and cancer risk.
A strength of our study is the large size and the detailed information on pesticide use that allows for an assessment of individual pesticides in relation to the development of aggressive PCa. Pesticide applicators can reliably report on their use of pesticides [51, 52]. We found the reported duration of pesticide use by applicators showed a high level of agreement with the duration of plausible market availability across all pesticides. In addition, we focused our analyses to clinically relevant subtypes of $\mathrm{PCa}$ to identify risk factors for this more aggressive subtype of disease. When we evaluated the possible influence of PSA screening on risk estimates, we found that use of individual pesticides associated with risk was not related to a history of PSA screening (eliminating potential biases due to screening).

There were also limitations. The ability to evaluate cumulative lifetime use for some pesticides first reported in Phase 2 and Phase 3 is limited which may result in non-differential exposure misclassification. However, this exposure misclassification would likely result in biasing our results toward the null [53]. Moreover, this is not an issue for the pesticides reported in Table 2, where lifetime use before enrollment was assessed. We were also not able to conduct exposure-response analyses for all users of a given pesticide because lifetime duration of use information was only ascertained during the Phase 3 questionnaire. And in some instances, duration of use for pesticides that were reported had small numbers for adequately powered exposure-response analysis. For many of the pesticides reported in Table 3, registration dates are relatively close to the time of prostate cancer diagnosis (approximately 5-15 years). Although there are limited data on the latent period for prostate cancer, it is possible that insufficient time has lapsed between exposure and cancer development for many of the pesticides evaluated here. In fact, thus far, positive associations for prostate cancer have only been observed for insecticides that were first registered for use primarily in the 1940s-1960s, suggesting a long latent period for pesticide-induced prostate cancer. Finally, we also made several comparisons, increasing the likelihood of a chance finding.

\section{Conclusion}

We found a significantly elevated risk of aggressive $\mathrm{PCa}$ among ever users of the OP insecticide dimethoate and an inverse association between ever use of the herbicide triclopyr. The association we observed between dimethoate and aggressive $\mathrm{PCa}$ adds another dithioate insecticide to the list of OPs associated with PCa risk in the AHS. Few other pesticides were associated with aggressive $\mathrm{PCa}$, but extended follow-up of the cohort is warranted for this long latency cancer. 


\section{Supplementary information}

Supplementary information accompanies this paper at https://doi.org/10. 1186/s12940-020-00583-0.

Additional file 1: Table S1. Association between pesticide (ever/never use) and overall PCa for those pesticides first reported at the Phase 1 take-home (TH) questionnaire in the Agricultural Health Study (AHS).

Additional file 2: Table S2. Association between pesticide (ever/never use) and overall PCa for those pesticides first reported at follow-up (Phase 2 and Phase 3 questionnaires) in the Agricultural Health Study (AHS).

Additional file 3: Table S3. Correlation analysis between take-home pesticides and organophosphates previously identified as associated with aggressive PCa in the AHS

Additional file 4: Table S4. Selected characteristics of participants in the Agricultural Health Study (AHS) cohort; aggressive prostate cancer (PCa) cases reported through 2014 in North Carolina and 2015 in lowa ${ }^{a}$.

Additional file 5: Table S5. Prostate specific antigen (PSA) frequencies by selected characteristics ${ }^{\mathrm{a}}$.

\section{Abbreviations}

AHS: Agricultural Health Study; BMI: Body mass index; CATI: Computer assisted telephone interview; Cl: Confidence interval; EPA: Environmental Protection Agency; HR: Hazard ratio; IARC: International Agency for Research on Cancer; ICD-O-3: International Classification of Diseases for Oncology, third revision; OC: Organochlorine; OP: Organophosphate; PCa: Prostate cancer; PSA: Prostate specific antigen; RED: Registration Eligibility Decision

\section{Acknowledgements}

Not applicable

\section{Authors' contributions}

SK, LEBF conceptualized the research question. SK, LEBF, JNH, CGP, DPS, AB, GA were involved in the design and acquisition of the data. LAP and SK analyzed the data and drafted the manuscript. All authors (CCL, LEBF, SK, GA, $J \mathrm{NH}, \mathrm{CGP}, \mathrm{DPS}, \mathrm{JHL}, \mathrm{AB}, \mathrm{LAP}$ ) provided interpretation of the results and the written manuscript. All authors have read and approved the final manuscript.

\section{Funding}

This work was supported [in part] by the intramural research program of the National Institutes of Health, the National Institute of Environmental Health Sciences (Z01-ES049030) and National Cancer Institute (Z01-CP010119).

\section{Availability of data and materials}

The Agricultural Health Study has procedures in place for access of study data that can be found at (https://aghealth.nih.gov/collaboration/process.html).

\section{Ethics approval and consent to participate}

The study protocol, including implied informed consent for completion of questionnaires, was approved by all relevant institutional review boards.

\section{Consent for publication}

Not applicable

\section{Competing interests}

The authors declare that they have no competing interests.

\section{Author details}

${ }^{1}$ Occupational and Environmental Epidemiology Branch, Division of Cancer Epidemiology and Genetics, National Cancer Institute, 9609 Medical Center Drive, Rockville, MD 20850, USA. ${ }^{2}$ National Institute of Environmental Health Sciences, 111 T.W. Alexander Drive, Research Triangle Park, NC 27709, USA. ${ }^{3}$ Biostatistics Branch, Division of Cancer Epidemiology and Genetics, National Cancer Institute, 9609 Medical Center Drive, Rockville, MD 20850, USA ${ }^{4}$ Occupational and Environmental Epidemiology Branch, Division of Cancer Epidemiology and Genetics, National Cancer Institute, 9609 Medical Center Dr., Room \#6E616, MSC 9771, Bethesda, MD 20892, USA.
Received: 13 November 2019 Accepted: 20 February 2020

Published online: 05 March 2020

\section{References}

1. Tangen CM, Heuhouser ML, Stanford JL. Prostate cancer. In: Thun MJ, Linet MS, Cerhan C, Haiman C, Schottenfeld D, editors. Schottenfeld and Fraumeni Cancer Epidemiology and Prevention. 4th ed. New York: Oxford University Press; 2018. p. 1128-50.

2. Jahn JL, Giovannucci EL, Stampfer MJ. The high prevalence of undiagnosed prostate cancer at autopsy: implications for epidemiology and treatment of prostate cancer in the prostate-specific antigen-era. Int J Cancer. 2015: 137(12):2795-802

3. Parent ME, Desy M, Siemiatycki J. Does exposure to agricultural chemicals increase the risk of prostate cancer among farmers? Mcgill J Med. 2009; 12(1):70-7.

4. Blair A, Dosemeci M, Heineman EF. Cancer and other causes of death among male and female farmers from twenty-three states. Am J Ind Med. 1993:23(5):729-42.

5. Blair A, Zahm SH, Pearce NE, Heineman EF, Fraumeni JF Jr. Clues to cancer etiology from studies of farmers. Scand J Work Environ Health. 1992;18(4): 209-15

6. Dosemeci M, Hoover RN, Blair A, et al. Farming and prostate cancer among African-Americans in the southeastern United States. J Natl Cancer Inst. 1994:86(22):1718-9.

7. Lemarchand $C$, Tual S, Boulanger $M$, et al. Prostate cancer risk among French farmers in the AGRICAN cohort. Scand J Work Environ Health. 2016; 42(2):144-52.

8. Meyer TE, Coker AL, Sanderson M, Symanski E. A case-control study of farming and prostate cancer in African-American and Caucasian men. Occup Environ Med. 2007;64(3):155-60.

9. Van Maele-Fabry G, Willems JL. Prostate cancer among pesticide applicators: a meta-analysis. Int Arch Occup Environ Health. 2004;77(8):559-70.

10. Lerro CC, Koutros S, Andreotti G, et al. Cancer incidence in the agricultural health study after 20 years of follow-up. Cancer Causes Control. 2019;30(4): 311-22

11. Srishti Shresthaa CGP, Keilb AP, Umbachc DM, Lerrod CC, Lynche CF, Chenf $H$, Blaird A, Koutrosd S, Hofmannd JN, Beane Freemand LE, Sandler DP. Mortality in the agricultural health study: an updated analysis through; 2015.

12. Koutros S, Beane Freeman LE, Lubin JH, et al. Risk of total and aggressive prostate cancer and pesticide use in the agricultural health study. Am J Epidemiol. 2013:177(1):59-74.

13. Koutros $\mathrm{S}$, Langseth $\mathrm{H}$, Grimsrud TK, et al. Prediagnostic serum organochlorine concentrations and metastatic prostate cancer: a nested case-control study in the Norwegian Janus Serum Bank Cohort. Environ Health Perspect. 2015;123(9):867-72.

14. Kumar V, Yadav CS, Singh S, et al. CYP $1 A 1$ polymorphism and organochlorine pesticides levels in the etiology of prostate cancer. Chemosphere. 2010;81(4):464-8.

15. Multigner L, Ndong JR, Giusti A, et al. Chlordecone exposure and risk of prostate cancer. J Clin Oncol. 2010;28(21):3457-62.

16. Emeville E, Giusti A, Coumoul X, Thome JP, Blanchet P, Multigner L. Associations of plasma concentrations of dichlorodiphenyldichloroethylene and polychlorinated biphenyls with prostate cancer: a case-control study in Guadeloupe (French West Indies). Environ Health Perspect. 2015:123(4):317-23.

17. Alavanja MC, Sandler DP, McMaster SB, et al. The agricultural health study. Environ Health Perspect. 1996;104(4):362-9.

18. Fritz AG. International classification of diseases for oncology: ICD-O. Geneva: World Health Organization; 2000.

19. U.S. Environmental Protection Agency (EPA). Chemicals registered for the first time as pesticidal active ingredients under FIFRA. Washington, DC: Office of Pesticide Programs; 1994.

20. Farm chemicals handbook. Willoughby: Meister Pub. Co.; 2001.

21. Cooperative Extension Offices of Cornell University, Michigan State University, Oregon State University, and University of California at Davis. http://pmep.cce.cornell.edu/profiles/extoxnet/. Accessed 28 Feb 2019.

22. Pohanish RP, Sittig M. Sittig's handbook of pesticides and agricultural chemicals. 2nd ed. Norwich.: William Andrew Publishing; 2015.

23. U.S. Environmental Protection Agency (EPA). Fluazifop-P-butyl. Report of the Metabolism Assessment Review Committee. Washington, D.C: Office of Prevention PaTS, ed; 2004 
24. U.S. Environmental Protection Agency (EPA). Federal register notice: disulfoton and methamidophos; product cancellation order In: Agency USEP, ed. Vol 74 2009:48551-48554.

25. Centers for Disease Control and Prevention (CDC). Biomonitoring summary: dimethoate. 2016; https://www.cdc.gov/biomonitoring/ DimethoateOmethoate BiomonitoringSummary.html.

26. International Agency for Research on Cancer (IARC). Miscellaneous pesticides. IARC Monogr Eval Carcinog Risks Hum. 1983;30:409-424.

27. Reuber MD. Carcinogenicity of dimethoate. Environ Res. 1984;34(2):193-211.

28. U.S. Department of Agriculture. The biologic and economic assessment of dimethoate. 1979. https://naldc.nal.usda.gov/download/CAT81766128/PDF.

29. Band PR, Abanto Z, Bert J, et al. Prostate cancer risk and exposure to pesticides in British Columbia farmers. Prostate. 2011;71(2):168-83.

30. Schumacher FR, Al Olama AA, Berndt SI, et al. Association analyses of more than 140,000 men identify 63 new prostate cancer susceptibility loci. Nat Genet. 2018;50(7):928-36.

31. Koutros S, Berndt SI, Hughes Barry K, et al. Genetic susceptibility loci, pesticide exposure and prostate cancer risk. PLoS One. 2013;8(4):e58195.

32. U.S. Environmental Protection Agency (EPA). Reregistration Eligibility Decision (RED): Triclopyr. 1998.

33. Extension Toxicology Network Pesticide Information Profiles (EXTOXNET). Triclopyr; 1993. http://pmep.cce.cornell.edu/profiles/extoxnet/pyrethrinsziram/triclopyr-ext.html. Accessed 28 Feb 2019.

34. Meeker JD, Ravi SR, Barr DB, Hauser R. Circulating estradiol in men is inversely related to urinary metabolites of nonpersistent insecticides. Reprod Toxicol. 2008;25(2):184-91.

35. Meeker JD, Ryan L, Barr DB, Hauser R. Exposure to nonpersistent insecticides and male reproductive hormones. Epidemiology. 2006;17(1):61-8.

36. Fortenberry GZ, Hu H, Turyk M, Barr DB, Meeker JD. Association between urinary 3, 5, 6-trichloro-2-pyridinol, a metabolite of chlorpyrifos and chlorpyrifos-methyl, and serum T4 and TSH in NHANES 1999-2002. Sci Total Environ. 2012;424:351-5.

37. Mondul AM, Weinstein SJ, Bosworth T, Remaley AT, Virtamo J, Albanes D. Circulating thyroxine, thyroid-stimulating hormone, and hypothyroid status and the risk of prostate cancer. PLoS One. 2012;7(10):e47730.

38. Mondul AM, Moore SC, Weinstein SJ, Karoly ED, Sampson JN, Albanes D. Metabolomic analysis of prostate cancer risk in a prospective cohort: the alpha-tocolpherol, beta-carotene cancer prevention (ATBC) study. Int J Cancer. 2015;137(9):2124-32.

39. Hellevik Al, Asvold BO, Bjoro T, Romundstad PR, Nilsen TI, Vatten LJ. Thyroid function and cancer risk: a prospective population study. Cancer Epidemiol Biomark Prev. 2009;18(2):570-4.

40. Hoption Cann SA, Qiu Z, van Netten C. A prospective study of iodine status, thyroid function, and prostate cancer risk: follow-up of the first national health and nutrition examination survey. Nutr Cancer. 2007;58(1):28-34.

41. Hsieh ML, Juang HH. Cell growth effects of triiodothyronine and expression of thyroid hormone receptor in prostate carcinoma cells. J Androl. 2005; 26(3):422-8.

42. Lehrer S, Diamond EJ, Stone NN, Droller MJ, Stock RG. Serum triiodothyronine is increased in men with prostate cancer and benign prostatic hyperplasia. J Urol. 2002;168(6):2431-3.

43. Tsui $\mathrm{KH}, \mathrm{Hsieh}$ WC, Lin MH, Chang PL, Juang HH. Triiodothyronine modulates cell proliferation of human prostatic carcinoma cells by downregulation of the B-cell translocation gene 2. Prostate. 2008;68(6):610-9.

44. Carmichael NG, Nolan RJ, Perkins JM, Davies R, Warrington SJ. Oral and dermal pharmacokinetics of triclopyr in human volunteers. Hum Toxicol. 1989;8(6):431-7

45. Goldner WS, Sandler DP, Yu F, et al. Hypothyroidism and pesticide use among male private pesticide applicators in the agricultural health study. J Occup Environ Med. 2013;55(10):1171-8.

46. International Agency for Research on Cancer (IARC). DDT, Lindane, and 2,4D. In: IARC Monographs on the Evaluation of Carcinogenic Risks to Humans; 2018. p. 113.

47. U.S. Environmental Protection Agency (EPA). Reregistration Eligibility Decision (RED): Bromoxynil. In: Agency USEP, ed. 1998.

48. U.S. Environmental Protection Agency (EPA). Reregistration Eligibility Decision (RED): Linuron. 1995.

49. Bai J, Han H, Wang F, et al. Maternal linuron exposure alters testicular development in male offspring rats at the whole genome level. Toxicology. 2017;389:13-20
50. U.S. Environmental Protection Agency (EPA). Reregistration Eligibility Decision (RED): Sethoxydim. In: Agency USEP, ed. 2005.

51. Blair A, Tarone R, Sandler D, et al. Reliability of reporting on life-style and agricultural factors by a sample of participants in the agricultural health study from lowa. Epidemiology. 2002;13(1):94-9.

52. Hoppin JA, Yucel F, Dosemeci M, Sandler DP. Accuracy of self-reported pesticide use duration information from licensed pesticide applicators in the agricultural health study. J Expo Anal Environ Epidemiol. 2002;12(5):313-8.

53. Blair A, Thomas K, Coble J, et al. Impact of pesticide exposure misclassification on estimates of relative risks in the agricultural health study. Occup Environ Med. 2011;68(7):537-41.

\section{Publisher's Note}

Springer Nature remains neutral with regard to jurisdictional claims in published maps and institutional affiliations.
Ready to submit your research? Choose BMC and benefit from:

- fast, convenient online submission

- thorough peer review by experienced researchers in your field

- rapid publication on acceptance

- support for research data, including large and complex data types

- gold Open Access which fosters wider collaboration and increased citations

- maximum visibility for your research: over $100 \mathrm{M}$ website views per year

At BMC, research is always in progress.

Learn more biomedcentral.com/submissions 